\title{
An X-Ray Diffraction Study on Amorphous Transition Metal-Boron Alloys Obtained by Different Preparation Methods
}

\author{
Anna Corrias, Guido Ennas, Giorgio Paschina, and Daniela Zedda \\ Dipartimento di Scienze Chimiche, Via Ospedale 72, I-09124 Cagliari \\ Z. Naturforsch. 50a, 672-676 (1995); received Feburary 20, 1995
}

\begin{abstract}
The metal-metal structure of amorphous metal-boron alloys prepared by melt spinning, chemical reduction and mechanical alloying is compared. The general features of the structure functions and radial distribution functions obtained from X-ray diffraction data are similar. The first peak of the radial distribution function, mainly due to metal-metal nearest neighbours, has been simulated by a peak shape function. In all the investigated samples the best fit was obtained using two slightly different metal-metal distances, which suggests the presence of an asymmetric distribution.
\end{abstract}

Key words: Amorphous Alloys X-ray Diffraction Powders.

\section{Introduction}

Amorphous TM-B (TM = Transition Metal) alloys for structural investigations have mostly been prepared by the Melt Spinning (MS), based on rapid quenching. Other preparation methods have been developed, particularly those producing amorphous alloy powders, which represent the most suitable form for the compaction into tridimensional objects for technologicl applications [1, 2]. Chemical Reduction (CR) of metal ions by potassium or sodium borohydride in aqueous solutions [3,4] and Mechanical Alloying (MA) of elemental powders $[5,6]$ have also proven to be suitable to this end.

Despite the great deal of experimental work, a comparison of the structural characteristics of amorphous alloys obtained by different methods has only occasionally been carried out $[7,8]$.

We present the results of an X-ray diffraction study of amorphous TM-B alloys obtained by MS, CR, and MA. The analysis of X-ray diffraction data can be conveniently used to study TM-TM correlations in TM-B systems, as the boron scattering factor is much weaker than that of the metal. The analysis was carried out despite the different composition of some of the amorphous alloys: in fact the different mechanism of formation has not allowed to obtain alloys of equal composition in the used preparation methods.

Reprint requests to Dr. Anna Corrias (Fax. -39-70-669272).

\section{Experimental}

Three samples of $\mathrm{TM}-\mathrm{B}(\mathrm{TM}=\mathrm{Fe}, \mathrm{Co}, \mathrm{Ni})$ alloys in form of very fine powders were prepared by reducing $\mathrm{Fe}^{2+}, \mathrm{Co}^{2+}$ and $\mathrm{Ni}^{2+}$ ions in aqueous solution using $\mathrm{KBH}_{4}$ [4]. The compositions of the samples, which is not easy to adjust because of the many parameters which influence the process [9], are close to $\mathrm{TM}_{60} \mathrm{~B}_{40}$. The $\mathrm{Co}-\mathrm{B}$ and $\mathrm{Ni}-\mathrm{B}$ samples are completely amorphous while the $\mathrm{Fe}-\mathrm{B}$ sample contains a small fraction of $\alpha-\mathrm{Fe}$ crystallites. There is a small amount of oxygen present in the form of a layer of boron oxide on the particle surface $[4,10]$ which protects the highly pyrophoric powders.

A sample of $\mathrm{Co}_{60} \mathrm{~B}_{40}$ was prepared by milling pure Co (Ventron $99.8 \%$ ) and B (Ventron 99.7\%) powders in a Fritsch Pulverisette 5 ball mill. Milling was performed under an argon atmosphere using stainless steel balls and vials. The sample mainly consisted of an amorphous phase accompanied by traces of fcc Co [6]. On the other hand, in $\mathrm{Ni}-\mathrm{B}$ and $\mathrm{Fe}-\mathrm{B}$ systems the amorphization reaction did not proceed significantly [11].

Three ribbons of amorphous $\mathrm{TM}-\mathrm{B}(\mathrm{TM}=\mathrm{Fe}, \mathrm{Co}$, $\mathrm{Ni}$ ) alloys were prepared using a Buhler 7400 melt spinning apparatus working with a wheel rate of $35 \mathrm{~m} / \mathrm{s}$ at $80 \mathrm{mbar}$ argon overpressure; a quartz crucible with $1 \mathrm{~mm}$ hole was used. The final thickness of the ribbons was approximately $30 \mu \mathrm{m}$. Only the $\mathrm{Ni}-\mathrm{B}$ sample could be prepared with the $\mathrm{Ni}_{60} \mathrm{~B}_{40}$ composition because this system is easily melt spun 
over a wide range of $\mathrm{B}$ content [12]. The other two samples could only be prepared with the composition $\mathrm{TM}_{80} \mathrm{~B}_{20}$, corresponding to their best glass forming ability.

Structure functions and radial distribution functions were calculated from X-ray intensity data collected on a Siemens $\theta-2 \theta$ diffractometer equipped with a graphite monochromator. Diffracted intensities were collected at preset points in the angular range $4^{\circ}<2 \theta<140^{\circ}$ corresponding to $0.6<s<16.6 \AA^{-1}$ ( $s=4 \pi \sin \theta / \lambda, \operatorname{MoK} \alpha$ radiation). At least 10000 counts per step were collected using a narrow scanning step $\left(\Delta 2 \theta=0.2^{\circ}\right)$ in the angular range $4^{\circ}<2 \theta<80^{\circ}$, and at least 40000 counts in the range $80^{\circ}<2 \theta<140^{\circ}$ with a scanning step of $\Delta 2 \theta=0.5^{\circ}$.

The diffracted intensities were corrected for background, absorption and polarisation, and were normalized to electronic units by the high angle method [13]. From the normalized intensities, $I_{\text {eu }}$, the structure functions, $i(s)$, were obtained according to

$$
i(s)=I_{\mathrm{eu}}-\sum_{i} n_{i} f_{i}^{2}(s),
$$

where $n_{i}$ are the stoichiometric coefficients of the assumed unit and $f_{i}(s)$ are the scattering factors of the species $i$. The reduced radial distribution functions $Q(r)$ were obtained by Fourier transformation:

$$
Q(r)=(2 / \pi) \int_{0}^{s_{\max }} s i(s) M(s) \sin (s r) \mathrm{d} s,
$$

where $M(s)$ is a modification function of the form

$$
M(s)=\left[\sum n_{i} f_{i}(0)^{2} / \sum n_{i} f_{i}(s)^{2}\right] \exp \left(-0.005 s^{2}\right) .
$$

$n_{i}=1$ was assigned to the TM atom. A correction for spurious peaks below $1.5 \AA$ was also applied in the radial distribution function [13].

To obtain quantitative information about the first coordination shell, the first peak of the radial distribution functions was simulated using a peak shape function. Theoretical peaks were calculated by Fourier transforming the pair contributions to the structure functions:

$$
\left(2-\delta_{i j}\right) n_{i} f_{i} N_{i j}\left(\sin s r_{i j} / s r_{i j}\right) \exp \left(-0.5 \sigma_{i j}^{2} s^{2}\right),
$$

where $\delta$ is the Kronecker function, $r_{i j}$ is the average distance between atoms $i$ and $j, \sigma_{i j}$ is the associated root mean squares deviation and $N_{i j}$ is the average number of $j$ atoms surrounding an $i$ atom.

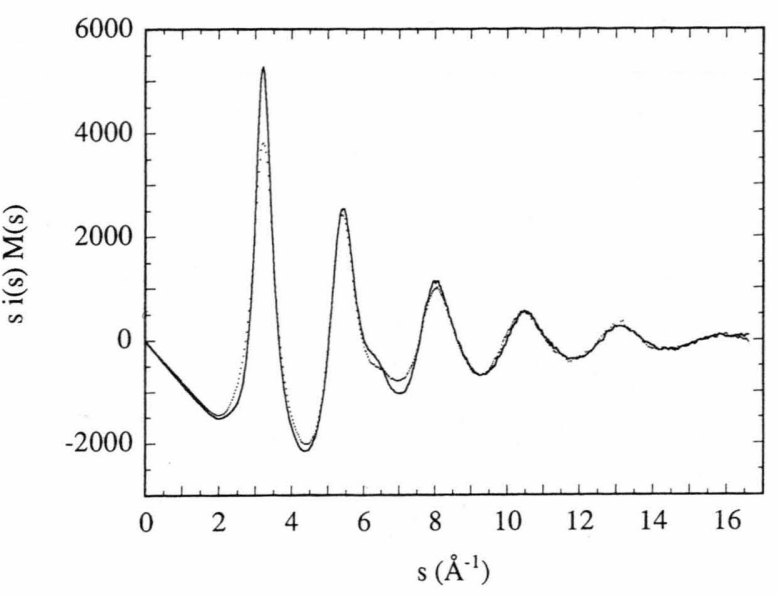

Fig. 1. X-ray structure functions for $\mathrm{MS}-\mathrm{Ni}_{60} \mathrm{~B}_{40}$, continuous line, and $\mathrm{CR}-\mathrm{Ni}_{60} \mathrm{~B}_{40}$, dotted line.

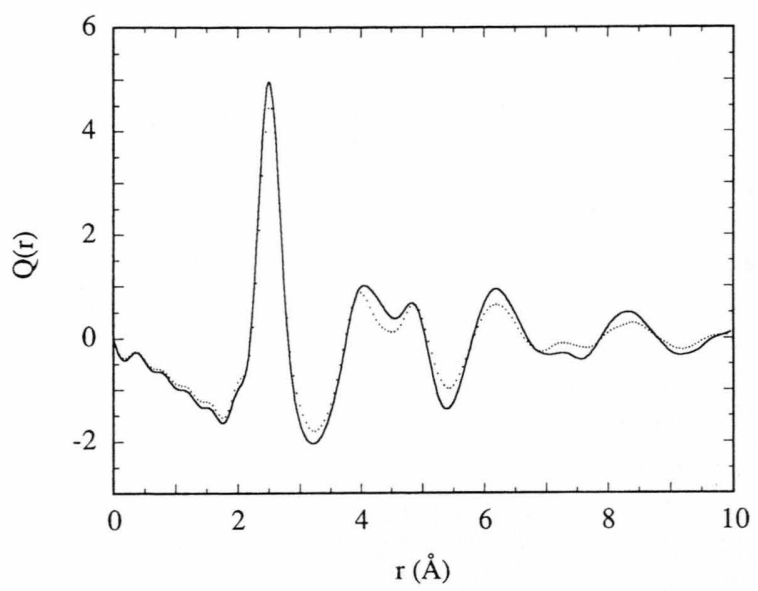

Fig. 2. X-ray radial distribution functions for $\mathrm{MS}-\mathrm{Ni}_{60} \mathrm{~B}_{40}$, continuous line, and $\mathrm{CR}-\mathrm{Ni}_{60} \mathrm{~B}_{40}$, dotted line.

\section{Results}

Figures 1 and 2 show the structure functions, si(s) $M(s)$, and the corresponding radial distribution functions, $Q(r)$, for $\mathrm{CR}-\mathrm{Ni}_{60} \mathrm{~B}_{40}$ and $\mathrm{MS}-\mathrm{Ni}_{60} \mathrm{~B}_{40}$ samples. Both functions are similar for the two samples. Minor differences can be noticed in the first oscillations of the structure functions, which are slightly broader for the CR sample than for the MS one. The $Q(r)$ peaks are also slightly broader for the CR sample than for the MS sample, and the difference becomes more evident with increasing $r$. 


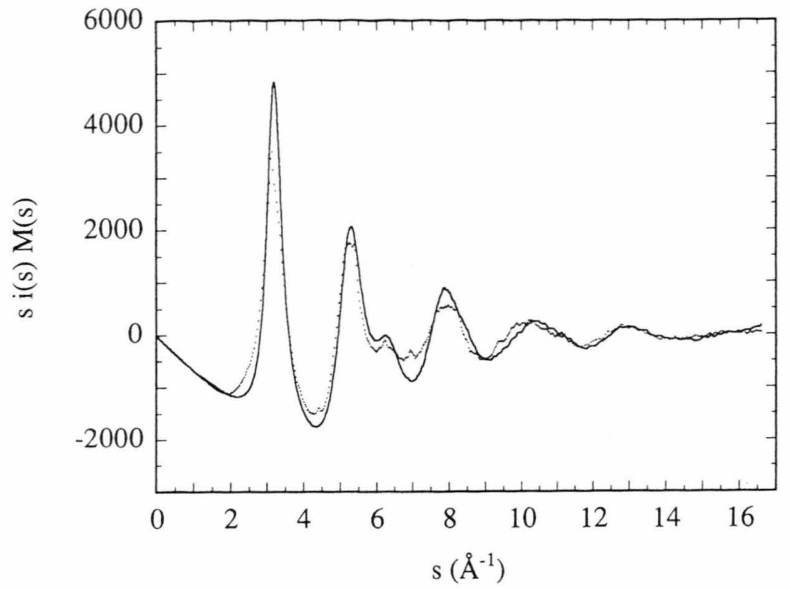

Fig. 3. X-ray structure functions for MS- $\mathrm{Fe}_{80} \mathrm{~B}_{20}$, continuous line, and $\mathrm{CR}-\mathrm{Fe}_{60} \mathrm{~B}_{40}$, dotted line.

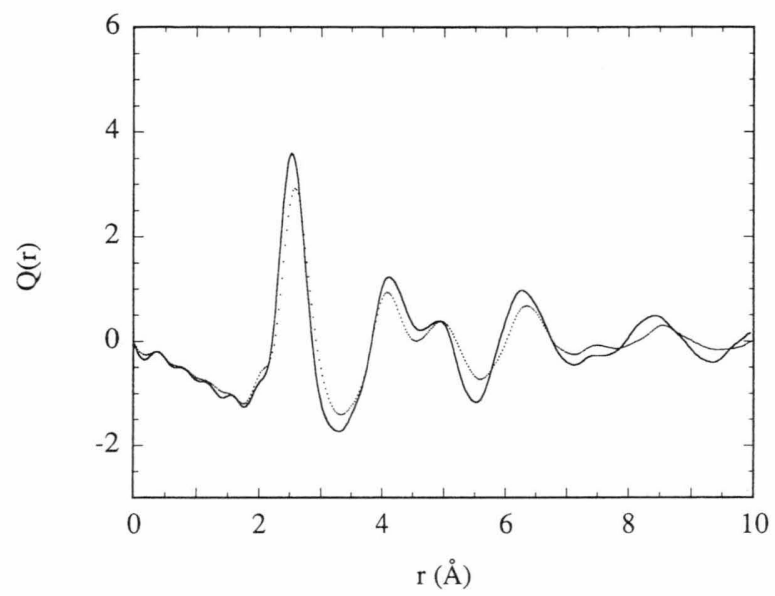

Fig. 4. X-ray radial distribution functions for $\mathrm{MS}-\mathrm{Fe}_{80} \mathrm{~B}_{20}$, continuous line, and $\mathrm{CR}-\mathrm{Fe}_{60} \mathrm{~B}_{40}$, dotted line.

Figures 3 and 4 show the structure functions and the corresponding radial distribution functions for the MS $-\mathrm{Fe}_{80} \mathrm{~B}_{20}$ and $\mathrm{CR}-\mathrm{Fe}_{60} \mathrm{~B}_{40}$ samples. In this case some differences exist between the two samples, certainly due to their different composition. The oscillations at high $s$ of the two structure functions are slightly out of phase. Moreover the presence of some crystallites of $\alpha$-Fe in the CR sample gives rise to small peaks in its structure function.

Figures 5 and 6 show the structure functions and the corresponding radial distribution functions for the $\mathrm{MS}-\mathrm{Co}_{80} \mathrm{~B}_{20}, \mathrm{CR}-\mathrm{Co}_{60} \mathrm{~B}_{40}$ and $\mathrm{MA}-\mathrm{Co}_{60} \mathrm{~B}_{40}$ sam-

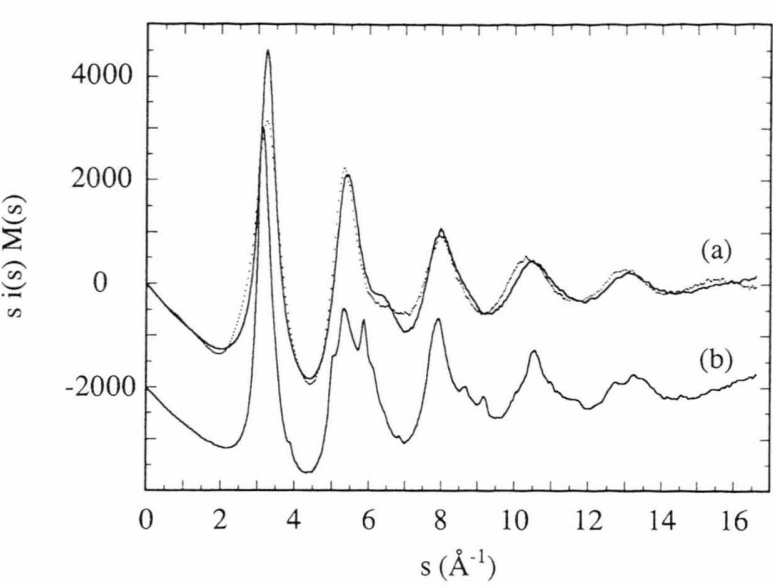

Fig. 5. X-ray structure functions for (a) $\mathrm{MS}-\mathrm{Co}_{80} \mathrm{~B}_{20}$, continuous line, and $\mathrm{CR}-\mathrm{Fe}_{60} \mathrm{~B}_{40}$, dotted line, and (b) MA$\mathrm{Co}_{60} \mathrm{~B}_{40}$.

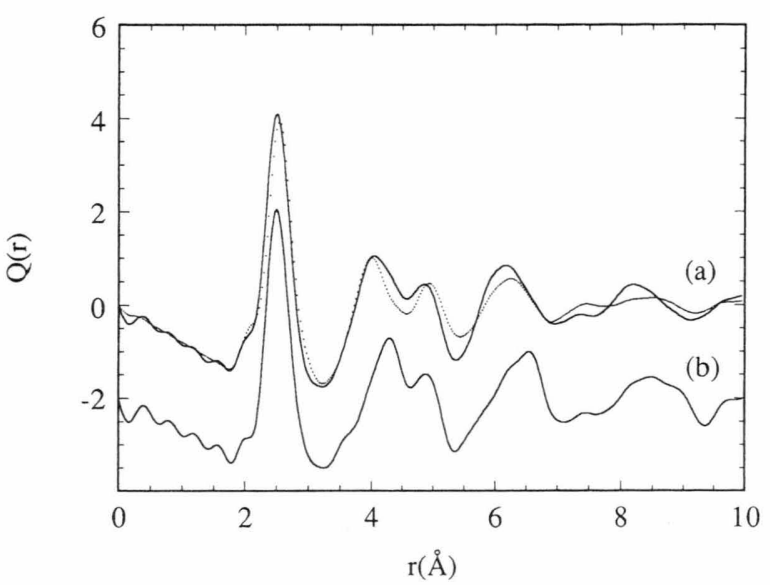

Fig. 6. X-ray radial cistribution functions for (a) MS$\mathrm{Co}_{80} \mathrm{~B}_{20}$, continuous line, and $\mathrm{CR}-\mathrm{Fe}_{60} \mathrm{~B}_{40}$, dotted line, and (b) $\mathrm{MA}-\mathrm{Co}_{60} \mathrm{~B}_{40}$.

ples. The oscillations of the structure functions and the peaks of the radial distribution functions are broader for the CR sample than for the MS one, as already observed for the $\mathrm{Ni}-\mathrm{B}$ and $\mathrm{Fe}-\mathrm{B}$ samples; the oscillations at high $s$ in the structure functions are also slightly out of phase. In the MA sample traces of fcc Co crystallites, which have not completely reacted during the ball milling process, are detectable.

The first peak of the radial distribution functions of all the samples is centred around $2.5 \AA$ and is mainly due to TM-TM nearest neighbours; despite the small scattering power of boron, a shoulder around $2 \AA$ due 
Table 1. Distances, $r$, coordination numbers, $N$ and root mean square deviations, $\sigma$, obtained by peak shape analysis.

\begin{tabular}{lllllrl}
\hline Sample & $r(\AA)$ & $\sigma(\AA)$ & $N$ & $r(\AA)$ & $\sigma(\AA)$ & $N$ \\
\hline $\mathrm{MS}-\mathrm{Ni}_{60} \mathrm{~B}_{40}$ & $2.50 \pm 0.01$ & $0.13 \pm 0.01$ & $7.9 \pm 0.8$ & $2.78 \pm 0.01$ & $0.18 \pm 0.01$ & $2.8 \pm 0.3$ \\
$\mathrm{CR}-\mathrm{Ni}_{60} \mathrm{~B}_{40}$ & $2.51 \pm 0.01$ & $0.12 \pm 0.01$ & $6.8 \pm 0.7$ & $2.78 \pm 0.01$ & $0.17 \pm 0.01$ & $2.4 \pm 0.2$ \\
$\mathrm{MS}-\mathrm{Fe}_{80} \mathrm{~B}_{20}$ & $2.50 \pm 0.01$ & $0.13 \pm 0.01$ & $6.8 \pm 0.7$ & $2.76 \pm 0.01$ & $0.17 \pm 0.01$ & $3.4 \pm 0.3$ \\
$\mathrm{CR}-\mathrm{Fe}_{60} \mathrm{~B}_{40}$ & $2.55 \pm 0.01$ & $0.11 \pm 0.01$ & $6.5 \pm 0.7$ & $2.88 \pm 0.01$ & $0.18 \pm 0.01$ & $3.1 \pm 0.3$ \\
$\mathrm{MS}-\mathrm{Co}_{80} \mathrm{~B}_{20}$ & $2.49 \pm 0.01$ & $0.13 \pm 0.01$ & $7.5 \pm 0.7$ & $2.76 \pm 0.01$ & $0.17 \pm 0.01$ & $2.4 \pm 0.2$ \\
$\mathrm{CR}-\mathrm{Co}_{60} \mathrm{~B}_{40}$ & $2.52 \pm 0.01$ & $0.13 \pm 0.01$ & $6.8 \pm 0.7$ & $2.79 \pm 0.01$ & $0.17 \pm 0.01$ & $2.5 \pm 0.2$ \\
$\mathrm{MA}-\mathrm{Co}_{60} \mathrm{~B}_{40}$ & $2.49 \pm 0.01$ & $0.11 \pm 0.01$ & $6.6 \pm 0.7$ & $2.79 \pm 0.01$ & $0.13 \pm 0.01$ & $2.3 \pm 0.2$ \\
\hline
\end{tabular}

to TM-B nearest neighbours is present. The first peak of the radial distribution functions has been fitted using a peak shape function by refining coordination numbers, distances and mean squares deviations for TM-TM interactions. The best fit was obtained when TM-TM contributions with different numbers of atoms at two different distances were introduced. The resulting structural parameters are reported in Table 1; TM-B parameters were also introduced in the fitting procedure, but they are not reported because of their large uncertainty due to the small weighting factor of the TM-B correlations.

\section{Discussion}

X-ray diffraction data on TM-B alloys are dominated by TM-TM correlations. The general features of the X-ray structure functions and radial distribution functions of amorphous TM-B alloys, prepared using different techniques, show that the TM-TM structuring is similar in all the samples.

The CR and $\mathrm{MS} \mathrm{Ni}{ }_{60} \mathrm{~B}_{40}$ samples present the closest resemblance. The similarity between these two samples has also been confirmed by the shapes of boron $\mathrm{K}$-edges and nickel L-edges obtained by electron energy loss spectroscopy (EELS) [10]. Some minor differences can be observed: the fine CR powder shows broader oscillations than the MS sample in the low $s$ region of the structure function $\operatorname{si}(s)$, while the high $s$ part is perfectly coincident. This is also consistent with the fact that $Q(r)$ peaks tend to become smaller and broader as $r$ increases; in fact the high $s$ region of the structure function is dominated by the nearest neighbour correlations while the low $s$ part is also due to correlations at larger distances. The TMTM distances and root mean squares deviations obtained from the peak shape analysis are coincident within experimental error for the two samples; however the slightly smaller coordination numbers ob- tained for the CR sample seem meaningful. In fact, the smaller coordination numbers together with the broadening of $Q(r)$ with increasing $r$ seem to be due to the higher surface/volume ratio of the fine powders where the atoms on or near the surface contribute less and less to the radial distribution function as $r$ increases.

The structure functions of $\mathrm{CR}-\mathrm{Fe}_{60} \mathrm{~B}_{40}$ and $\mathrm{CR}-$ $\mathrm{Co}_{60} \mathrm{~B}_{40}$ are slightly out of phase in the high $s$ region in comparison with the corresponding curves of MS$\mathrm{Fe}_{80} \mathrm{~B}_{20}$ and $\mathrm{MS}-\mathrm{Co}_{80} \mathrm{~B}_{20}$, in agreement with the longer first TM-TM distances in the CR samples; in fact the high $s$ part of the structure function is dominated by the TM-TM nearest neighbour distances. The longer distance seems due to the larger boron content of the CR samples as already found in TM-B amorphous alloys of different compositions $[14,15]$. Moreover, some faint differences appear between the $\mathrm{CR}-\mathrm{Fe}_{60} \mathrm{~B}_{40}$ and $\mathrm{MS}-\mathrm{Fe}_{80} \mathrm{~B}_{20}$ structure and radial distribution functions, due to the presence of some $\alpha-\mathrm{Fe}$ crystallites in the CR sample. The presence of a crystalline component is more evident in MA$\mathrm{Co}_{60} \mathrm{~B}_{40}$.

It must be pointed out that an overall similarity of the TM-TM structure in all the samples is observed, despite the differences in composition and/or the presence of a small crystalline component in some samples.

The structure of amorphous metal-metalloid alloys prepared by MS has been extensively studied by means of diffraction and absorption techniques [16]. EXAFS results, characterised by shorter distances, were interpreted as due to the presence of an asymmetric nearest neighbour distance distribution [17]. Other authors claimed that, if an asymmetric distribution is present, this information should also be provided by scattering measurements [18]. An asymmetric TM-TM first peak was actually observed in melt spun $\mathrm{Ni}_{64} \mathrm{~B}_{36}$ by neutron scattering under high spatial resolution [19]. 
In the present study, when the first peak of the radial distribution functions was fitted using only one TM-TM contribution (and one TM-B contribution), the difference between the experimental curve and the theoretical peak shape clearly showed an extra contribution, centred on the right hand side of the peak. Therefore a good fit was only obtained by introducing two different TM-TM distances with a large number of neighbours at a shorter distance and a lower number at a longer distance; this is a clear evidence of the presence of an asymmetric distribution of TM-TM distances. The asymmetric distribution of distances has been observed in all the samples, confirming the similarity of TM-TM structure in amorphous TM-B alloys obtained using different preparation methods.

\section{Conclusions}

The TM-TM structure of amorphous metal-boron alloys obtained using different preparation routes has

[1] F. E. Luborsky (ed.), Amorphous Metallic Alloys, Butterworths, London 1983.

[2] R.W. Cahn (ed.), Materials Science and Technology: A Comprehensive Treatment, Vol. IX, Chapter 9, VCH, Weinheim, Germany 1991.

[3] J. Van Wonterghem, S. Mørup, C. J.W. Kock, S. W. Charles, and S. Wells, Nature London 322, 622 (1986).

[4] A. Corrias, G. Ennas, G. Marongiu, A. Musinu, and G. Paschina, Chem. Mater. 5, 1722 (1993).

[5] A. Corrias, G. Ennas, G. Marongiu, A. Musinu, and G. Paschina, J. Mater. Res. 8, 1327 (1993).

[6] A. Corrias, G. Ennas, G. Marongiu, A. Musinu, and G. Paschina, J. Non-Cryst. Solids 163, 35 (1993).

[7] W. M. Kuschke, L. Schultz, P. Lamparter, and S. Steeb, Z. Naturforsch. 46a, 491 (1991).

[8] F. Buffa, A. Corrias, G. Licheri, G. Navarra, and D. Raoux, J. Non-Cryst. Solids 151, 119 (1992).

[9] G. N. Glavee, K. J. Klabunde, C. M. Sorensen, and G. C. Hadjipanayis, Langmuir 9, 162 (1993).

[10] G. Mountjoy, A. Corrias, and P. H. Gaskell, J. NonCryst. Solids, submitted. been studied by X-ray diffraction. The general features of the structure functions and radial distribution functions of the alloys prepared by different methods are similar. The small differences can be related to the different morphologies of the material. In fact, powder presents a much higher surface/volume ratio. The peak shape analysis of the first main peak of the radial distribution functions show that an asymmetric distribution of TM-TM distances is present in all the investigated samples.

\section{Acknowledgements}

The authors would like to thank Dr. Franco Padella for assistance with using the Melt Spinning apparatus. The financial support of CNR and MURST is gratefully acknowledged.

[11] A. Corrias, G. Ennas, G. Marongiu, A. Musinu, G. Paschina, and D. Zedda, Mater. Sci. Eng., in press.

[12] A. Inoue, A. Kitamura, and T. Masumoto, J. Mater. Sci. 16, 1895 (1981).

[13] M. Magini (ed.), X-ray Diffraction of Ions in Aqueous Solutions: Hydration and Complex Formation, CRC, Boca Raton, Florida 1988.

[14] G. S. Chadha, N. Cowlam, H. A. Davies, and I. W. Donald, J. Non-Cryst. Solids 44, 265 (1981).

[15] K. Suzuki, T. Fukunaga, F. Itoh, and N. Watanabe, in: S. Steeb and H. Warliamont (eds.), Rapidly Quenched Metals, Elsevier Science Publishers, Amsterdam 1985, pp. $479-482$.

[16] P. H. Gaskell, in: H. Beck and H. J. Güntherodt (eds.), Glassy Metals II, Springer-Verlag, Berlin 1983, pp. 5-50.

[17] M. De Crescenzi, A. Balzarotti, F. Comin, L. Incoccia, S. Mobilio, and M. Motta, Solid State Commun. 37, 921 (1981).

[18] G. S. Cargill III, J. Non-Cryst. Solids 61 \& 62, 261 (1984).

[19] P. P. Gardner, N. Cowlam, and H. A. Davies, J. Phys. F 15, 769 (1985). 